\title{
From isolated to networked: a paradigmatic shift in mitochondrial physiology
}

\section{Miguel A. Aon*}

Johns Hopkins University, School of Medicine, Institute of Molecular Cardiobiology, Baltimore, MD, USA

\section{Edited by:}

Paul S. Brookes, University of

Rochester, USA

\section{Reviewed by:}

Valdur Saks, National Institute of Chemical Physics and Biophysics,

Estonia

Florian Muller, University of Texas Health Science Center at San Antonio, USA

Paul S. Brookes, University of

Rochester, USA

\section{*Correspondence:}

Miguel A. Aon, Institute of Molecular

Cardiobiology, School of Medicine,

Johns Hopkins University, 720 Rutland

Avenue, Ross Building 1059, Baltimore,

MD 21205, USA.

e-mail:maon1@jhmi.edu
A new paradigm of mitochondrial function in networks is emerging which includes, without undermining, the glorious and still useful paradigm of the isolated mitochondrion. The mitochondrial network paradigm introduces new concepts, tools, and analytical techniques. Among them is that mitochondrial function in networks exhibits interdependence and multiplicative effects based on synchronization mechanisms, which involve communication between mitochondrial neighbors. The collective dynamics of these networks become advantageous for coordinating function spanning from the cell, to the tissue, and the organ. However, under severely stressful conditions the network behavior of mitochondria may become life threatening.

Keywords: emergent network properties, systems biology, reactive oxygen species, collective dynamics, energy supply and demand
It has become almost common place in the biological literature to refer to the influential work of the science historian and epistemologist Tomas Kuhn in order to analyze paradigmatic shifts in science (Strohman, 1997; Mayr, 2004). For Kuhn, a paradigm is comprised by conceptual schemes or models (e.g., Copernican, Mendelian, Darwinian) which are utilized by the majority, in a particular scientific community. Scientific work within a paradigm allows its reinforcement and as such is essentially conservative; Kuhn dubbed it "normal science". However, the constant practice of a paradigm shows its weaknesses until a point in which they cannot be ignored anymore. This is the point where "paradigm shifts" happen. The thesis of this article is that the field of mitochondrial physiology is at this point now.

The new paradigmatic shift starts to emerge when dust is not yet settled for mitochondria expanding role from powerhouse to signaling organelle, allowing them to gather the reputation of "gatekeepers of life and death" (better than the "housekeeper" notion attributed to them, and metabolism in general, during the heydays of molecular biology). The respectable but insufficient paradigm of the isolated mitochondrion with which every mitochondrial physiologist has grown up, starts to be replaced - without denying it - by the more realistic paradigm of mitochondrial function in networks. This paradigm shift in mitochondrial physiology echoes the more general return to integrative physiology, now called Systems Biology. A more complete understanding of networks and their properties is a key to understanding the conceptual idea driving this paradigm shift (Barabasi and Oltvai, 2004; Weiss et al., 2006; Aon et al., 2007).

The paradigm of the isolated mitochondrion was very much guided by the description of its biochemical machinery - of which the respiratory chain and the tricarboxylic acid (TCA) cycle represent major achievements - and the chemiosmotic principle introduced by Peter Mitchell. An illustration of what this paradigm is unable to explain is that, under critical conditions, a failure in the mitochondrial network can take your life if it happens in your heart and, I would like to anticipate, in your brain. Mitochondrial function in a network is inter-dependent resulting in multiplicative rather than additive effects. Under normal physiological conditions, the coordinated function of mitochondria confers robustness (i.e., a failure in a few of them does not affect overall function) whereas under pathophysiological conditions failure in a considerable number of mitochondria is needed to initiate the collapse of the whole network (Aon et al., 2004; Zhou et al., 2010). For example, the triggering of the apoptotic pathway by cytochrome $c$ release, or the opening of sarcolemmal $\mathrm{K}_{\text {ATP }}$ channels under energy failure, do not depend on one or just a few mitochondria but on a considerable number of them. The collapse of the mitochondrial network membrane potential under oxidative stress involves at least $60 \%$ of them, representing an emergent, macroscopic, property of the network (Aon et al., 2004). Emergent properties, the single most distinguishing feature of complex behavior in a system, cannot be anticipated from the behavior of the isolated components of such a system. The statistical behavior of the mitochondrial network, consistent with fractal, scale-free, oscillatory dynamics (Aon et al., 2006; Beraud et al., 2009, see Glossary), represent an emergent behavior in the form of dynamic fractals. In the case of mitochondria, dynamic fractals express the long-term correlation (memory) and intrinsic coordination of the mitochondrial network dynamics, which does not exhibit a "characteristic" oscillatory frequency but a broad range of them. The existence of dynamic fractals can be of basic significance for the 
scale-free temporal organization of energetic timekeeping, from the organelle to the cell, and organism levels, and across evolutionary scales (Aon et al., 2008b; Lloyd and Rossi, 2008).

In the emerging paradigm, mitochondria represent "hubs" in the network of metabolic processes because the TCA cycle sits at the convergence of most catabolic and anabolic pathways (Aon et al., 2007). This positioning of the TCA cycle gives mitochondria, as a network within the more general metabolic network, the character of a true "hub" due to their multiple links to other pathways, as either an input (source) or an output (sink).

Morphologically, mitochondria form dynamic networks, regular - lattice-like as in the heart or skeletal muscle - or irregular - reticular-like as in neurons. The functional and morphological dynamic behavior of these networks largely influence as well as reflect the status of the entire cellular, tissue, and organ, ensemble (Benard and Rossignol, 2008). Highly non-linear mechanisms govern the network dynamics, highlighting the inseparability of the spatial and temporal aspects of mitochondrial network behavior, under both physiological and pathophysiological conditions. The strong interconnectedness among components bestows scaling properties to these networks, implying that the result of an action in the subcellular realm, e.g., mitochondrial network, can escalate to the tissue and organ levels, such as under crises, like heart attack or disease, resulting in catastrophic arrhythmias (Aon et al., 2009). The propagation of mitochondrial failure under critical conditions is amplified by the multiple non-linear interactions among electrical, mechanical, and energetic, processes (Zhou et al., 2009; Yang et al., 2010).

It is timely to stress at this point that the dynamic of mitochondrial networks does not necessarily imply physical continuity, for chemical communication among mitochondrial neighbors can convey information about their status. In fact, local diffusion ( 1-2 $\mu \mathrm{m}$ between mitochondrial neighbors) of chemical messengers such as ROS, can give long-range $(\sim 100 \mu \mathrm{m}$ cellular, or even longer tissue and organ), global, behavior (Aon et al., 2004; Zhou et al., 2010). The physiological status of the global network, gathered through local interactions, is the macroscopic spatial cue of the network collective dynamics. In heart, the collective dynamics in key energetic and redox mitochondrial variables $-\Delta \Psi_{\mathrm{m}}, \mathrm{NADH}$, and ROS - is oscillatory. Under physiological conditions, the oscillatory dynamics is of low amplitude and high frequency, depending on a loose, weak, ROS communication. Under stressful conditions, such as lack of substrate or oxidative stress (Romashko et al., 1998; Aon et al., 2003), ROS becomes a strong coupling messenger resulting in low frequency, high-amplitude, cell-wide oscillations of the mitochondrial network (Aon et al., 2008a).

The tight match between energy supply and demand in the heart can be more readily fulfilled by the highly synchronized and robust action of mitochondrial networks. In the heart cell, during maximal workload, the whole ATP pool can be turned over in a few seconds since $\sim 2 \%$ of that pool is consumed in each heart beat (Harris and Das, 1991). From a temporal perspective, these numbers underscore the highly tuned response in energy supply by the mitochondrial network to the demand by electro-mechanical events operating in the millisecond range (e.g., action potential, calcium transient, myofibrils crossbridge cycling) (Cortassa et al., 2006). The changing metabolic demand as a function of workload requires both constancy and flexibility in mitochondrial functionality; constancy to provide a steady supply of ATP to fuel contraction, and flexibility, to adapt the rate of respiration and mitochondrial ATP production.

The notion of mitochondrial networks within networks, i.e., the overall metabolic network, stresses the fact that not all the control of mitochondrial respiration in vivo, resides within the mitochondrion itself. In fact, as part of the network of mechano-electrical processes in the heart, mitochondria, as the main energy suppliers, both modulate and are modulated by the overall network. For instance, during the contraction cycle, when the contractile force is close to its maximum, the respiratory flux is not only controlled by mitochondrial processes but also by the cytoplasmic and sarcolemmal ATP-consuming motors and pumps, like the myofibrillar and $\mathrm{Na} / \mathrm{K}$ ATPases (Cortassa et al., 2009).

\section{A PROSPECTIVE FOR FUTURE STUDIES WITHIN THE PARADIGM OF MITOCHONDRIAL NETWORKS}

The study of mitochondrial function as and in networks implies considering unique traits such as interdependence, multiplicative effects, collective dynamics spanning several temporal scales, and communication. Together, these traits are necessary and sufficient to define the confines, conceptually and in tools, of a new paradigm.

Several attempts, whose approach qualifies for the network paradigm, are to a certain extent already underway in the brain (Nicholls, 2008) and in the heart (Aon et al., 2008a,b; Arsac et al., 2008; Beraud et al., 2009; Zhou et al., 2009, 2010; Yang et al., 2010). Applying this integrative approach to cells is the most immediate use of the new paradigm, which includes but transcends by far the mere analysis of differences between isolated and in vivo mitochondrial physiology.

Simulating complex physiological responses with computational models, at several simultaneous temporal and spatial scales (Cortassa et al., 2006; Shim et al., 2006; Plank et al., 2008; Cortassa et al., 2009), in conjunction with high throughput technologies, has a great future for its potential ability to produce unprecedented insights into the inner working of organ physiological mechanisms, under health and disease. The combined experimentalcomputational strategy represents the edge of the future for mitochondrial networks. The computational approach provides the ability to calculate basic control and regulatory properties of extended networks, comprising processes of different nature, i.e., metabolic, transport, electro-mechanical, like in the heart. Due to the interdependence factor that characterizes network behavior, affecting them with pharmacological agents, triggers diffuse control loops with consequences that go beyond intuition and the expected targets, i.e., so-called "secondary" or "collateral" effects (see Cortassa et al., 2009, and Glossary).

The emerging science of networks represents a new chapter within systemic approaches to Biology and Medicine in health and disease. Mitochondrial physiology already belongs to this new era.

\section{ACKNOWLEDGMENTS}

The author gratefully acknowledges Dr. Sonia Cortassa for critically reading the manuscript. This work was supported by NIH grants R01-HL091923-01 and P01-HL081427. 


\section{GLOSSARY}

Network: The collective organization of an ensemble of objects, or groups of them, in space (structural and topological) and time (dynamics).

Emergent behavior: Novel and sometimes surprising macroscopic properties arising from the self-organizing capacity of biological systems' internal structure and dynamics. Self-organization appears in non-linear open systems like cells, organisms, and ecosystems, away from thermodynamic equilibrium, i.e., constantly driven by exchange of matter and energy or information with the environment.

Fractal: An object of geometric, statistical, or dynamical nature that obeys power laws of the form $M(L) \propto L^{D}$, with $D$ as the non-integer fractal dimension ( $\propto$ means "is proportional to"). Mandelbrot introduced "fractal geometry" and defined a fractal "as a shape made of parts similar to the whole in some way". This last property is called self-similarity, and represents one of the most distinctive features of fractals.

Dynamic fractals: Self-similar statistical objects described in time series, i.e., the time-dependent behavior of an observable (e.g., mitochondrial membrane potential, NADH). These

\section{REFERENCES}

Aon, M.A., Cortassa, S., Akar, F. G., Brown, D. A., Zhou, L., and O'Rourke, B. (2009). From mitochondrial dynamics to arrhythmias. Int. J. Biochem. Cell Biol. 41, 1940-1948.

Aon, M. A., Cortassa, S., Marban, E., and O'Rourke, B. (2003). Synchronized whole cell oscillations in mitochondrial metabolism triggered by a local release of reactive oxygen species in cardiac myocytes. J. Biol. Chem. 278, $44735-44744$.

Aon, M. A., Cortassa, S., and O'Rourke, B. (2004). Percolation and criticality in a mitochondrial network. Proc. Natl. Acad. Sci. U.S.A. 101, 4447-4452.

Aon, M.A., Cortassa, S., and O'Rourke, B. (2006). The fundamental organization of cardiac mitochondria as a network of coupled oscillators. Biophys. J. 91, 4317-4327.

Aon, M. A., Cortassa, S., and O'Rourke, B. (2007). On the Network Properties of Mitochondria. Weinheim: Wiley-VCH Verlag GmbH \& Co. KGaA.

Aon, M. A., Cortassa, S., and O'Rourke, B. (2008a). Mitochondrial oscillations in physiology and pathophysiology. $A d v$. Exp. Med. Biol. 641, 98-117.

Aon, M. A., Roussel, M. R., Cortassa, S., O'Rourke, B., Murray, D. B., Beckmann, M., and Lloyd, D. (2008b). The scale-free dynamics of eukaryotic cells. PLoS One 3, e3624. doi:10.1371/ journal.pone.000362.

Arsac, L. M., Beuste, C., Miraux, S., Deschodt-Arsac, V., Thiaudiere, E., Franconi, J. M. and Diolez, P. H.
(2008). In vivo modular control analysis of energy metabolism in contracting skeletal muscle. Biochem. J. 414, 391-397.

Barabasi, A. L., and Oltvai, Z. N. (2004). Network biology: understanding the cell's functional organization. Nat. Rev. Genet. 5, 101-113.

Benard, G., and Rossignol, R. (2008). Ultrastructure of the mitochondrion and its bearing on function and bioenergetics. Antioxid. Redox Signal. 10, 1313-1342.

Beraud, N., Pelloux, S., Usson, Y., Kuznetsov, A. V., Ronot, X., Tourneur, Y., and Saks, V. (2009). Mitochondrial dynamics in heart cells: very low amplitude high frequency fluctuamotion in non beating $\mathrm{Hl}-1$ cells. J. Bioenerg. Biomembr. 41, 195-214.

Cortassa, S., Aon, M. A., O'Rourke, B., Jacques, R., Tseng, H. J., Marban, E., and Winslow, R. L. (2006). A computational model integrating electrophysiology, contraction, and mitochondrial bioenergetics in the ventricular myocyte. Biophys. J. 91, 1564-1589.

Cortassa, S., O’Rourke, B., Winslow, R. L., and Aon, M.A. (2009). Control and regulation of mitochondrial energetics in an integrated model of cardiomyocyte function. Biophys. J. 96, 2466-2478.

Harris, D. A., and Das, A. M. (1991). Control of mitochondrial ATP synthesis in the heart. Biochem. J. 280 (Pt 3), 561-573.

Lloyd,D., and Rossi,E. L. (2008)."Epilogue: a new vision of life," in Ultradian tions in adult cardiomyocytes and flow

statistical fractals exhibit scale-free dynamics, i.e., they span a wide range of temporal scales simultaneously (e.g., from milliseconds to several minutes), in such a way that the short-term fluctuations are related to longer-term trends in the dynamic behavior. As a result, what affects one time scale affects them all: a fundamental property of dynamic fractals. The long-term correlation in mitochondrial dynamic behavior can be interpreted as memory since their status in the present is dependent on their condition in the past, a fact underscored by their non-random statistical behavior.

Control by diffuse loops: It is defined as the control that a process A exerts over process $\mathrm{C}$ or $\mathrm{D}$ without an apparent direct mechanistic link among them. This is a new conceptual tool uncovered from calculating control and regulatory properties of the overall network of energetic and electro-mechanical processes involved in cardiomyocyte function. For example, inhibition of the $\mathrm{Na} / \mathrm{K}$ ATPase controls mitochondrial respiration through an extensive diffuse loop involving the sarcoplasmic reticulum $\mathrm{Ca}^{2+}$ ATPase (SERCA), the sarcolemmal Na$/ \mathrm{Ca}^{2+}$ exchanger, and the levels of cytoplasmic $\mathrm{Na}^{+}, \mathrm{Ca}^{2+}$, and ATP.

Rhythms from Molecules to Mind, eds D. Lloyd and E. Rossi (New York: Springer Science + Business Media BV), 431-439.

Mayr, E. (2004). What Makes Biology Unique? New York: Cambridge University Press.

Nicholls, D. G. (2008). Oxidative stress and energy crises in neuronal dysfunction. Ann. N.Y. Acad. Sci. 1147, 53-60.

Plank, G., Zhou, L., Greenstein, J. L. Cortassa, S., Winslow, R. L., O’Rourke, B., and Trayanova, N. A. (2008). From mitochondrial ion channels to arrhythmias in the heart: computational techniques to bridge the spatio-temporal scales. Phil. Trans. 366, 3381-3409.

Romashko, D. N., Marban, E., and O'Rourke, B. (1998). Subcellular metabolic transients and mitochondrial redox waves in heart cells. Proc. Natl. Acad. Sci. U.S.A. 95, 1618-1623.

Shim, E. B., Leem, C. H., Abe, Y., and Noma, A. (2006). A new multi-scale simulation model of the circulation: from cells to system. Phil. Trans. 364, 1483-1500.

Strohman, R. C. (1997). The coming Kuhnian revolution in biology. Nat. Biotechnol. 15, 194-200.

Weiss, J. N., Yang, L., and Qu, Z. (2006) Systems biology approaches to metabolic and cardiovascular disorders: network perspectives of cardiovascular metabolism. J. Lipid Res. 47, 2355-2366.

Yang, L., Korge, P., Weiss, J. N., and Qu, Z. (2010). Mitochondrial oscillations and waves in cardiac myocytes: insights from computational models. Biophys J. 98, 1428-1438.

Zhou, L., Aon, M. A., Almas, T., Cortassa, S., Winslow, R. L., and O'Rourke, B. (2010). A reaction-diffusion model of ROS-induced ROS release in a mitochondrial network. PLoS Comput. Biol.6, e1000657. doi:10.1371/journal. pcbi.1000657.

Zhou, L., Cortassa, S., Wei, A. C., Aon, M. A., Winslow, R. L., and O’Rourke, B. (2009). Modeling cardiac action potential shortening driven by oxidative stress-induced mitochondrial oscillations in guinea pig cardiomyocytes. Biophys. J. 97, 1843-1852.

Conflict of Interest Statement: The author declares that the research was conducted in the absence of any commercial or financial relationships that could be construed as a potential conflict of interest.

Received:09 May 2010; paperpending published:08 June 2010; accepted: 25 June 2010; published online: 14 July 2010.

Citation: Aon MA (2010) From isolated to networked: a paradigmatic shift in mitochondrial physiology. Front. Physio. 1:20. doi: 10.3389/fphys.2010.00020

This article was submitted to Frontiers in Mitochondrial Research, a specialty of Frontiers in Physiology.

Copyright (C) 2010 Aon. This is an openaccess article subject to an exclusive license agreement between the authors and the Frontiers Research Foundation, which permits unrestricted use, distribution, and reproduction in any medium, provided the original authors and source are credited. 